Pacific Journal of Mathematics

A COEFFICIENT INEQUALITY FOR FUNCTIONS OF
POSITIVE REAL PART WITH AN APPLICATION TO 


\title{
A COEFFICIENT INEQUALITY FOR FUNCTIONS OF POSITIVE REAL PART WITH AN APPLICATION TO MULTIVALENT FUNCTIONS
}

\section{Albert E. Livingston}

\begin{abstract}
We obtain sharp bounds on the magnitude of certain determinants, whose entries are the coefficients of a function of positive real part in the unit disk. These inequalities are used to solve a coefficient problem for a certain subclass of multivalent functions.
\end{abstract}

Introduction. Let $P(z)=c_{0}+c_{1} z+\cdots$ be analytic in $\Delta=\{z$ : $|z|<1\}$ and satisfy $\operatorname{Re}(P(z))>0$ for $z$ in $\Delta$. The author [6] proved that the coefficients satisfy the inequality $\left|c_{n} / c_{0}-c_{1} c_{n-1} / c_{0}^{2}\right| \leq 2$ for all $n \geq 2$. This inequality was then used to obtain sharp bounds on the coefficients of functions in a subclass of multivalent close-to-convex functions. The inequality has recently been used by Libera and Zlotkiewicz [4] in their study of the coefficients of the inverses of convex functions. In \$2 of this paper we generalize the above inequality, obtaining precise bounds on the magnitude of certain determinants involving the coefficients $c_{n}$.

Section 3 of the paper deals with the coefficient problem for a certain class of multivalent functions. Goodman [1] has conjectured that if $f(z)=a_{1} z+\cdots$ is at most $p$-valent in $\Delta$ then for $n \geq p+1$,

$$
\left|a_{n}\right| \leq \sum_{t=1}^{p} \frac{2 t(n+p) !}{(p+t) !(p-t) !(n-p-1) !\left(n^{2}-t^{2}\right)}\left|a_{t}\right| .
$$

Inequality (1.1) reduces to the well-known Bieberbach conjecture when $p=1$. Let $S(p)$ be the class of functions which are analytic and $p$-valently starlike in $\Delta$. A function $f(z)$, analytic in $\Delta$ with $f(0)=0$, is a member of $S(p)$ if and only if there exists $\delta>0$ such that for $\delta<|z|<1$,

$$
\operatorname{Re}\left[\frac{z f^{\prime}(z)}{f(z)}\right]>0
$$

and for $\delta<r<1$,

$$
\int_{0}^{2 \pi} \operatorname{Re}\left[\frac{r e^{i \theta} f^{\prime}\left(r e^{i \theta}\right)}{f\left(r e^{i \theta}\right)}\right] d \theta=2 p \pi .
$$


We shall say that $F$ is in $K(p)$, if it is analytic in $\Delta$ and there exist $f$ in $S(p)$ and $\delta>0$ such that for $\delta<|z|<1$,

$$
\operatorname{Re}\left[\frac{z F^{\prime}(z)}{f(z)}\right]>0 \text {. }
$$

We shall say that $F$ is in $K(p)$ and analytic on $|z|=1$, if $F$ is analytic for $|z| \leq 1$ and there exist $f$, analytic for $|z| \leq 1$, such that (1.2), (1.3) and (1.4) hold for $|z|=1$. Inequality (1.1) was proved by Goodman and Robertson for a function in $S(p)$ in case all its coefficients are real [2] and by Robertson [7], if $a_{1}=a_{2}=\cdots=a_{p-2}=0$, the remaining coefficients being complex. The author [5] proved (1.1) for $n=p+1$ for functions in $K(p)$ and in [6] the inequality was proved for all $n \geq p+1$, if $f$ is in $K(p)$ and $a_{1}=a_{2}=\cdots=a_{p-2}=0$. A proof of (1.1) for functions in $K(p)$ with real coefficients appears in the literature [3]. However, the proof seems to be flawed (see for example MR 58 \#22521). In §3 we give a different and simpler proof of this result using the inequalities obtained in $\$ 2$.

\section{Coefficients of functions with positive real part.}

LEMMA 1. Let $\operatorname{Re} c_{0}>0$ and for $z$ in $\Delta$,

$$
P(z)=\left(\operatorname{Re} c_{0}\right) \sum_{j=1}^{m} \lambda_{j} \frac{1+e^{i t_{j} z}}{1-e^{i t_{j} z}}+i \operatorname{Im} c_{0}=\sum_{n=0}^{\infty} c_{n} z^{n},
$$

where for $j=1,2, \ldots, m, t_{j}$ and $\lambda_{j}$ are real with $\lambda_{j} \geq 0$ and $\sum_{j=1}^{m} \lambda_{j}=1$. Define for a fixed $n$ the $s$ by $s$ determinants $Q_{k}^{(s)}, s \geq 1, k=1,2, \ldots, m$ by

$$
Q_{k}^{(s)}=\left|\begin{array}{ccccc}
e^{i(n+s-2) t_{k}} & c_{n+s-2} / c_{0} & c_{n+s-3} / c_{0} & \cdots & c_{n} / c_{0} \\
1 & 1 & 0 & \cdots & 0 \\
e^{i t_{k}} & c_{1} / c_{0} & 1 & \cdots & 0 \\
e^{i 2 t_{k}} & c_{2} / c_{0} & c_{1} / c_{0} & \cdots & 0 \\
\vdots & \vdots & \vdots & & \vdots \\
e^{i(s-2) t_{k}} & c_{s-2} / c_{0} & c_{s-3} / c_{0} & \cdots & 1
\end{array}\right|
$$

then for $s=1,2, \ldots$ and $n=2,3, \ldots$

$$
\sum_{k=1}^{m} \lambda_{k}\left|Q_{k}^{(s)}\right|^{2}=1
$$


Proof. We have $c_{n}=\left(\operatorname{Re} c_{0}\right) \sum_{j=1}^{m} 2 \lambda_{j} e^{\mathrm{int},}$ for $n \geq 1$. Since a determinant is linear in any of its columns, we can write

$$
Q_{k}^{(s)}=\sum_{j=1}^{m} \lambda_{j}\left|\begin{array}{ccccc}
e^{i(n+s-2) t_{k}} & 2\left(\operatorname{Re} c_{0}\right) e^{i(n+s-2) t_{j}} / c_{0} & c_{n+s-3} / c_{0} & \cdots & c_{n} / c_{0} \\
1 & 1 & 0 & \cdots & 0 \\
e^{i t_{k}} & 2\left(\operatorname{Re} c_{0}\right) e^{i t_{j}} / c_{0} & 1 & \cdots & 0 \\
\vdots & \vdots & \vdots & & \vdots \\
e^{i(s-2) t_{k}} & 2\left(\operatorname{Re} c_{0}\right) e^{i(s-2) t_{j}} / c_{0} & c_{s-3} / c_{0} & \cdots & 1
\end{array}\right|
$$

Expanding the determinants by their second row we obtain

(2.2) $\quad Q_{k}^{(s)}=\left|\begin{array}{cccc}e^{i(n+s-2) t_{k}} & c_{n+s-3} / c_{0} & \cdots & c_{n} / c_{0} \\ e^{i t_{k}} & 1 & \cdots & 0 \\ e^{i 2 t_{k}} & c_{1} / c_{0} & \cdots & 0 \\ \vdots & \vdots & & \vdots \\ e^{i(s-2) t_{k}} & c_{s-3} / c_{0} & \cdots & 1\end{array}\right|$

$$
\begin{aligned}
& -\sum_{j=1}^{m} \frac{2\left(\operatorname{Re} c_{0}\right)}{c_{0}} \lambda_{j}\left|\begin{array}{cccc}
e^{i(n+s-2) t_{j}} & c_{n+s-3} / c_{0} & \cdots & c_{n} / c_{0} \\
e^{i t_{j}} & 1 & \cdots & 0 \\
e^{i 2 t_{j}} & c_{1} / c_{0} & \cdots & 0 \\
\vdots & \vdots & & \vdots \\
e^{i(s-2) t_{j}} & c_{s-3} / c_{0} & \cdots & 1
\end{array}\right| \\
& =e^{i t_{k}} Q_{k}^{(s-1)}-\frac{2\left(\operatorname{Re} c_{0}\right)}{c_{0}} \sum_{j=1}^{m} \lambda_{j} e^{i t_{j}} Q_{j}^{(s-1)}
\end{aligned}
$$

We proceed by induction. The case $s=1$ is trivial. For illustrative purposes we also consider the case $s=2 . Q_{k}^{(2)}=e^{\text {int }_{k}}-c_{n} / c_{0}$. Thus

$$
\begin{aligned}
\sum_{k=1}^{m} \lambda_{k}\left|Q_{k}^{(2)}\right|^{2} & =1-2 \sum_{k=1}^{m} \lambda_{k} \operatorname{Re}\left(e^{\left.-\mathrm{int}_{k} \frac{c_{n}}{c_{0}}\right)+\left|\frac{c_{n}}{c_{0}}\right|^{2}}\right. \\
& =1-2 \operatorname{Re} \sum_{k=1}^{m} \lambda_{k} e^{-\mathrm{int} t_{k}}\left(\frac{2 \operatorname{Re} c_{0}}{c_{0}}\right) \sum_{j=1}^{m} \lambda_{j} e^{\mathrm{int} j}+\left|\frac{c_{n}}{c_{0}}\right|^{2} \\
& =1-4 \operatorname{Re}\left(\frac{\operatorname{Re} c_{0}}{c_{0}}\right)\left|\sum_{j=1}^{m} \lambda_{j} e^{\mathrm{int}_{j}}\right|^{2}+\left|\frac{c_{n}}{c_{0}}\right|^{2} \\
& =1-\left|\frac{c_{n}}{c_{0}}\right|^{2}+\left|\frac{c_{n}}{c_{0}}\right|^{2}=1
\end{aligned}
$$


Assume that $\sum_{k=1}^{m} \lambda_{k}\left|Q_{k}^{(s-1)}\right|^{2}=1$ for some $s$. Using (2.2) we obtain,

$$
\begin{aligned}
& \sum_{k=1}^{m} \lambda_{k}\left|Q_{k}^{(s)}\right|^{2}=\sum_{k=1}^{m} \lambda_{k}\left|e^{i t_{k}} Q_{k}^{(s-1)}-\frac{2 \operatorname{Re} c_{0}}{c_{0}} \sum_{j=1}^{m} \lambda_{j} e^{i t_{j}} Q_{j}^{(s-1)}\right|^{2} \\
& =\sum_{k=1}^{m} \lambda_{k}\left|Q_{k}^{(s-1)}\right|^{2}-4 \operatorname{Re}\left(\frac{\operatorname{Re} c_{0}}{c_{0}}\right) \sum_{k=1}^{m} \lambda_{k} e^{-i t_{k}} \overline{Q_{k}^{(s-1)}} \sum_{j=1}^{m} \lambda_{j} e^{i t_{j}} Q_{j}^{(s-1)} \\
& \quad+4\left|\frac{\operatorname{Re} c_{0}}{c_{0}}\right|^{2}\left|\sum_{j=1}^{m} \lambda_{j} e^{i t} Q_{j}^{(s-1)}\right|^{2} \\
& =\sum_{k=1}^{m} \lambda_{k}\left|Q_{k}^{(s-1)}\right|^{2}+4\left[\left|\frac{\operatorname{Re} c_{0}}{c_{0}}\right|^{2}-\operatorname{Re}\left(\frac{\operatorname{Re} c_{0}}{c_{0}}\right)\right]\left|\sum_{j=1}^{m} \lambda_{j} e^{i t_{j}} Q_{j}^{(s-1)}\right|^{2} \\
& =\sum_{k=1}^{m} \lambda_{k}\left|Q_{k}^{(s-1)}\right|^{2}=1 .
\end{aligned}
$$

This then completes the proof of the lemma by induction.

THEOREM 1. Let $P(z)=c_{0}+c_{1} z+\cdots$ be analytic in $\Delta$ and satisfy $\operatorname{Re} P(z)>0$ for $z$ in $\Delta$. For $s=1,2,3, \ldots$ define the $(s+1)$ by $(s+1)$ determinant

$$
A_{n}^{(s)}=\left|\begin{array}{ccccc}
c_{n+s} / c_{0} & c_{n+s-1} / c_{0} & c_{n+s-2} / c_{0} & \cdots & c_{n} / c_{0} \\
c_{1} / c_{0} & 1 & 0 & \cdots & 0 \\
c_{2} / c_{0} & c_{1} / c_{0} & 1 & \cdots & 0 \\
\vdots & \vdots & \vdots & & \vdots \\
c_{s} / c_{0} & c_{s-1} / c_{0} & c_{s-2} / c_{0} & \cdots & 1
\end{array}\right| .
$$

Then for $s=1,2, \ldots$ and $n=1,2, \ldots$,

$$
\left|A_{n}^{(s)}\right| \leq 2\left|\frac{\operatorname{Re} c_{0}}{c_{0}}\right| \leq 2 .
$$

Equality is attained for $P(z)=(1+z) /(1-z)$.

Proof. Since functions satisfying the hypothesis of the theorem are uniform limits in compact subsets of $\Delta$ of functions of the form (2.1), we may restrict our consideration to such functions. Thus we may assume that the $c_{n}$ have the form

$$
c_{n}=\left(\operatorname{Re} c_{0}\right) \sum_{j=1}^{m} 2 \lambda_{j} e^{\mathrm{int},}
$$


We therefore have, using the linearity of $A_{n}^{(s)}$ in the first column

$$
\begin{aligned}
A_{n}^{(s)} & =\sum_{k=1}^{m} \frac{2 \operatorname{Re} c_{0}}{c_{0}} \lambda_{k} e^{i t_{k}}\left|\begin{array}{ccccc}
e^{i(n+s-1) t_{k}} & c_{n+s-1} / c_{0} & c_{n+s-2} / c_{0} & \cdots & c_{n} / c_{0} \\
1 & 1 & 0 & \cdots & 0 \\
e^{i t_{k}} & c_{1} / c_{0} & 1 & \cdots & 0 \\
\vdots & \vdots & \vdots & & \vdots \\
e^{i(s-1) t_{k}} & c_{s-1} / c_{0} & c_{s-2} / c_{0} & \cdots & 1
\end{array}\right| \\
& =\frac{2 \operatorname{Re} C_{0}}{C_{0}} \sum_{k=1}^{m} \lambda_{k} e^{i t_{k}} Q_{k}^{(s+1)} .
\end{aligned}
$$

Using the Schwarz inequality and the fact that $\sum_{k=1}^{m} \lambda_{k}=1$, we obtain

$$
\left|A_{n}^{(s)}\right|^{2} \leq\left|\frac{2 \operatorname{Re} C_{0}}{C_{0}}\right|^{2} \sum_{k=1}^{m} \lambda_{k}\left|Q_{k}^{(s+1)}\right|^{2}=\left|\frac{2 \operatorname{Re} C_{0}}{C_{0}}\right|^{2},
$$

by Lemma 1 . This gives the inequality stated in the theorem.

Corollary 1. Let $P(z)=c_{0}+c_{1} z+\cdots$ be analytic in $\Delta$ and satisfy $\operatorname{Re} P(z)>0$ for $z$ in $\Delta$ and let $1 / P(z)=d_{0}+d_{1} z+\cdots$, then for $p=$ $1,2, \ldots$,

$$
\left|\sum_{k=t}^{p} d_{k-t} c_{n-k}\right| \leq 2\left|\frac{\operatorname{Re} c_{0}}{c_{0}}\right| \leq 2
$$

for $t=0,1,2, \ldots, p$ and $n \geq p$.

Proof. Since $(P(z))(1 / P(z))=1$ we obtain for any $p=1,2, \ldots$ the system

$$
\begin{aligned}
& 1=d_{0} c_{0} \\
& 0=d_{0} c_{1}+d_{1} c_{0} \\
& 0=d_{0} c_{2}+d_{1} c_{1}+d_{2} c_{0} \\
& \vdots \\
& 0=d_{0} c_{p-t}+d_{1} c_{p-t-1}+\cdots+d_{p-t} c_{0} .
\end{aligned}
$$

Let

$$
B=\left|\begin{array}{cccc}
c_{0} & 0 & \cdots & 0 \\
c_{1} & c_{0} & \cdots & 0 \\
c_{2} & c_{1} & \cdots & 0 \\
\vdots & \vdots & & \vdots \\
c_{p-t} & c_{p-t-1} & \cdots & c_{0}
\end{array}\right|=c_{0}^{p-t+1}
$$


and let $B_{i j}$ be the minor of the element in the $i$ th row and $j$ th column of $B$. Using Cramer's rule we obtain for $j=0,1, \ldots,(p-t)$,

$$
d_{j}=\frac{(-1)^{j}}{c_{0}^{p-t+1}} B_{1, j+1} .
$$

Therefore in the notation of Theorem 1

$$
\begin{aligned}
\sum_{k=t}^{p} d_{k-t} c_{n-k}=\sum_{k=t}^{p} c_{n-k} \frac{(-1)^{k-t}}{c_{0}^{p-t+1}} B_{1, k-t+1} \\
=\frac{1}{c_{0}^{p-t+1}}\left|\begin{array}{cccc}
c_{n-t} & c_{n-t-1} & \cdots & c_{n-p} \\
c_{1} & c_{0} & \cdots & 0 \\
c_{2} & c_{1} & \cdots & 0 \\
\vdots & \vdots & & \vdots \\
c_{p-t} & c_{p-t+1} & \cdots & c_{0}
\end{array}\right|=A_{n-p}^{p-t} .
\end{aligned}
$$

Thus by Theorem 1

$$
\left|\sum_{k=t}^{p} d_{k-t} c_{n-k}\right|=\left|A_{n-p}^{p-t}\right| \leq 2\left|\frac{\operatorname{Re} c_{0}}{c_{0}}\right| \leq 2 .
$$

\section{Coefficients of functions in $K(p)$.}

LEMMA 2. For $n \geq p+1$ and $t=1,2, \ldots,(p-1)$

$$
\begin{aligned}
& 2+\frac{2 p(n+p) !}{(2 p) !(n-p-1) !\left(n^{2}-p^{2}\right)} \\
& \quad+\sum_{k=p+1}^{n-1} \frac{4 p(k+p) !}{(2 p) !(k-p-1) !\left(k^{2}-p^{2}\right)} \\
& =\frac{2 n(n+p) !}{(2 p) !(n-p-1) !\left(n^{2}-p^{2}\right)}, \\
& +\sum_{k=p+1}^{n-1}\left(\sum_{s=t+1}^{p} \frac{8 s(k+p) !}{(p+s) !(p-s) !(k-p-1) !\left(k^{2}-s^{2}\right)}\right) \\
& +\sum_{k=p+1}^{n-1} \frac{4 s(n+p) !}{(p+s) !(p-s) !(n-p-1) !\left(n^{2}-s^{2}\right)} \\
& +\frac{4 t(k+p) !}{(p+t) !(p-t) !(n-p-1) !\left(n^{2}-t^{2}\right)}
\end{aligned}
$$




$$
=\frac{2 n(n+p) !}{(p+t) !(p-t) !(n-p-1) !\left(n^{2}-t^{2}\right)} .
$$

If $n=p+1$, we interpret sums of the form $\sum_{k=p+1}^{n-1}$ as zero.

Proof. If $n=p+1$, both sides of (3.1) equal 2( $p+1)$. For $n \geq p+2$, (3.1) appears as equality (2.6) in [6]. If $n=p+1$ and $t=p-1$, both sides of (3.2) equal $(2 p+1)(p+1)$. For all $n \geq p+2$ and $t=p-1$, (3.2) appears as equality (2.5) in [6]. We assume then that (3.2) is true for a particular value of $t$ and all $n \geq p+1$. Replacing $t$ by $(t-1)$ in (3.2) and using the induction hypothesis we see that the proof of (3.2) will be complete if we can prove that for a fixed $t$ and all $n \geq p+1$,

$$
\begin{aligned}
& \frac{2(n+t)(n+p) !}{(p+t) !(p-t) !(n-p-1) !\left(n^{2}-t^{2}\right)} \\
& \quad+\frac{2(t-1)(n+p) !}{(p+t-1) !(p-t+1) !(n-p-1) !\left(n^{2}-(t-1)^{2}\right)} \\
& \quad+\sum_{k=p+1}^{n-1} \frac{4 t(k+p) !}{(p+t) !(p-t) !(k-p-1) !\left(k^{2}-t^{2}\right)} \\
& \quad+\sum_{k=p+1}^{n-1} \frac{4(t-1)(k+p) !}{(p+t-1) !(p-t+1) !(k-p-1) !\left(k^{2}-(t-1)^{2}\right)} \\
& =\frac{2 n(n+p) !}{(p+t-1) !(p-t+1) !(n-p-1) !\left(n^{2}-(t-1)^{2}\right)} .
\end{aligned}
$$

We will prove (3.3) by induction on $n$. If $n=p+1$, both sides of (3.3) equal $2(p+1)(2 p+1) !(p+t) !(p-t+2)$ !. Assume that (3.3) is true for a particular value of $n$. Replacing $n$ by $(n+1)$ and using the induction hypothesis, the left side of (3.3) becomes,

$$
\begin{aligned}
& \frac{2(n+t+1)(n+p+1) !}{(p+t) !(p-t) !(n-p) !\left((n+1)^{2}-t^{2}\right)} \\
& \quad+\frac{2(t-1)(n+p+1) !}{(p+t-1) !(p-t+1) !(n-p) !\left((n+1)^{2}-(t-1)^{2}\right)} \\
& +\frac{4 t(n+p) !}{(p+t) !(p-t) !(n-p-1) !\left(n^{2}-t^{2}\right)} \\
& +\frac{4(t-1)(n+p) !}{(p+t-1) !(p-t+1) !(n-p-1) !\left(n^{2}-(t-1)^{2}\right)}
\end{aligned}
$$




$$
\begin{aligned}
& 2 n(n+p) \text { ! } \\
& +\frac{2 n+t-1) !(p-t+1) !(n-p-1) !\left(n^{2}-(t-1)^{2}\right)}{(p+n)} \\
& -\frac{2(n+t)(n+p) !}{(p+t) !(p-t) !(n-p-1) !\left(n^{2}-t^{2}\right)} \\
& -\frac{2(t-1)(n+p) !}{(p+t-1) !(p-t+1) !(n-p-1) !\left(n^{2}-(t-1)^{2}\right)} \\
& =\frac{2(n+p+1) !}{(p+t) !(p-t) !(n-p) !(n-t+1)} \\
& +\frac{2(t-1)(n+p+1) !}{(p+t-1) !(p-t+1) !(n-p) !(n+t)(n-t+2)} \\
& -\frac{2(n+p) !}{(p+t) !(p-t) !(n-p-1) !(n+t)} \\
& +\frac{2(n+p) !}{(p+t-1) !(p-t+1) !(n-p-1) !(n-t+1)} \\
& =\frac{2(n+p) !}{(p+t) !(p-t) !(n-p-1) !}\left[\frac{n+p+1}{(n-p)(n-t+1)}-\frac{1}{n+t}\right] \\
& +\frac{2(n+p) !}{(p+t-1) !(p-t+1) !(n-p-1) !(n-t+1)} \\
& +\frac{2(t-1)(n+p+1) !}{(p+t-1) !(p-t+1) !(n-p) !(n+t)(n-t+2)} \\
& =\frac{2(2 n+1)(n+p) !}{(p+t-1) !(p-t) !(n-p) !(n-t+1)(n+t)} \\
& +\frac{2(n+p) !}{(p+t-1) !(p-t+1) !(n-p-1) !(n-t+1)} \\
& +\frac{2(t-1)(n+p+1) !}{(p+t-1) !(p-t+1) !(n-p) !(n+t)(n-t+2)} \\
& =\frac{2(n+p) !}{(p+t-1) !(p-t) !(n-p-1) !(n-t+1)} \\
& \times\left[\frac{2 n+1}{(n-p)(n+t)}+\frac{1}{(p-t+1)}\right] \\
& +\frac{2(t-1)(n+p+1) !}{(p+t-1) !(p-t+1) !(n-p) !(n+t)(n-t+2)}
\end{aligned}
$$




$$
\begin{aligned}
= & \frac{2(n+p+1) !}{(p+t-1) !(p-t+1) !(n-p) !(n+t)} \\
& +\frac{2(t-1)(n+p+1) !}{(p+t-1) !(p-t+1) !(n-p) !(n+t)(n-t+2)} \\
= & \frac{2(n+p+1) !}{(p+t-1) !(p-t+1) !(n-p) !(n+t)}\left[1+\frac{t-1}{n-t+2}\right] \\
= & \frac{2(n+1)(n+p+1) !}{(p+t-1) !(p-t+1) !(n-p) !\left((n+1)^{2}-(t-1)^{2}\right)}
\end{aligned}
$$

which is the right side of (3.3) with $n$ replaced by $(n+1)$. This completes the proof of (3.3) by induction.

LEMMA 3. Let $F(z)=a_{1} z+a_{2} z^{2}+\cdots$ be in $K(p)$ and be analytic for $|z| \leq 1$ with $a_{n}$ real for each $n$, then there exists $f(z)=b_{1} z+b_{2} z^{2}+\cdots$ in $S(p)$ and analytic for $|z| \leq 1$ with $b_{n}$ real for each $n$, such that $\operatorname{Re}\left(z F^{\prime}(z) / f(z)\right)>0$ for $|z| \leq 1$.

Proof. Since $F(z)$ is in $K(p)$ and analytic for $|z| \leq 1$, it is known that there exists $h(z)$ in $S(p)$ and analytic for $|z| \leq 1$ such that $\operatorname{Re} z F^{\prime}(z) / h(z)>0$ for $|z| \leq 1$. Let $P(z)=z F^{\prime}(z) / h(z)$ and $Q(z)=$ $[P(z) \overline{P(\bar{z})}]^{1 / 2}=\left[\left(z F^{\prime}(z)\right)^{2} / h(z) \overline{h(\bar{z})}\right]^{1 / 2}$, where we take the principal branch for the square root. Let $f(z)=z F^{\prime}(z) / Q(z)=b_{1} z+b_{2} z^{2}+\cdots$, then $f$ is analytic for $|z| \leq 1$ and $b_{n}$ is real for each $n$. Also $z f^{\prime}(z) / f(z)$ $=\frac{1}{2}\left(z h^{\prime}(z) / h(z)\right)+\frac{1}{2}\left(\bar{z} h^{\prime}(\bar{z}) / h(\bar{z})\right)$. Thus $\operatorname{Re} z f^{\prime}(z) / f(z)>0$ for $|z| \leq$ 1. Since $z F^{\prime}(z)$ has exactly $p$ zeros in $\Delta$ [5], $f(z)$ has exactly $p$ zeros in $\Delta$. Thus $f$ is in $S(p)$ and $\operatorname{Re}\left(z F^{\prime}(z) / f(z)\right)=\operatorname{Re} Q(z)>0$ for $|z| \leq 1$.

THEOREM 2. Let $F(z)=a_{1} z+a_{2} z^{2}+\cdots$ be in $K(p)$ with $a_{n}$ real for each $n$, then for $n \geq p+1$,

$$
\left|a_{n}\right| \leq \sum_{t=1}^{p} \frac{2 t(n+p) !}{(p+t) !(p-t) !(n-p-1) !\left(n^{2}-t^{2}\right)}\left|a_{t}\right| .
$$

Proof. Without loss of generality we may assume that $F$ is analytic for $|z| \leq 1$. Then by Lemma 3, there exists $f(z)=b_{1} z+b_{2} z^{2}+\cdots$ in $S(p)$ and analytic for $|z| \leq 1$ with $b_{n}$ real for each $n$, such that $\operatorname{Re}\left[z F^{\prime}(z) / f(z)\right]$ $>0$ for $|z| \leq 1$. Let $P(z)=z F^{\prime}(z) / f(z)=c_{0}+c_{1} z+\cdots$. Comparing coefficients, we obtain

$$
n a_{n}=\sum_{k=1}^{n} b_{k} c_{n-k}
$$


Let $1 / P(z)=d_{0}+d_{1} z+\cdots$. Comparing coefficients of both sides of the equality $f(z)=\left(z F^{\prime}(z)\right)(1 / P(z))$, we have,

$$
b_{n}=\sum_{t=1}^{n} t a_{t} d_{n-t}
$$

Combining (3.5) and (3.6), we have for $n \geq p+1$,

$$
\begin{aligned}
n a_{n} & =\sum_{k=1}^{p}\left(\sum_{t=1}^{k} t a_{t} d_{k-t}\right) c_{n-k}+\sum_{k=p+1}^{n} b_{k} c_{n-k} \\
& =\sum_{t=1}^{p}\left(\sum_{k=t}^{p} d_{k-t} c_{n-k}\right) t a_{t}+\sum_{k=p+1}^{n} b_{k} c_{n-k} .
\end{aligned}
$$

Making use of Corollary 1 and the fact that $\left|c_{n}\right| \leq 2\left|c_{0}\right|$, we obtain from (3.7)

$$
n\left|a_{n}\right| \leq \sum_{t=1}^{p} 2 t\left|a_{t}\right|+\sum_{k=p+1}^{n-1} 2\left|c_{0}\right|\left|b_{k}\right|+\left|c_{0}\right|\left|b_{n}\right|
$$

Since $f$ is in $S(p)$ and $b_{n}$ is real for all $n$, it is known [2] that for $k \geq p+1$,

$$
\left|b_{k}\right| \leq \sum_{s=1}^{p} \frac{2 s(k+p) !}{(p+s) !(p-s) !(k-p-1) !\left(k^{2}-s^{2}\right)}\left|b_{s}\right| .
$$

Since $\operatorname{Re}(1 / P(z))>0$ it follows that $\left|d_{n}\right| \leq 2\left|d_{0}\right|=2 /\left|c_{0}\right|$. Therefore, from (3.6) we have

$$
\left|b_{n}\right| \leq \sum_{t=1}^{n-1} \frac{2 t\left|a_{t}\right|}{\left|c_{0}\right|}+\frac{n\left|a_{n}\right|}{\left|c_{0}\right|}
$$

Combining (3.9) and (3.10) we have for $k \geq p+1$,

$$
\begin{aligned}
\left|b_{k}\right| \leq & \sum_{s=1}^{p} \frac{2 s(k+p) !}{(p+s) !(p-s) !(k-p-1) !\left(k^{2}-s^{2}\right)} \\
& \times\left(\sum_{t=1}^{s-1} \frac{2 t\left|a_{t}\right|}{\left|c_{0}\right|}+\frac{s\left|a_{s}\right|}{\left|c_{0}\right|}\right) .
\end{aligned}
$$


Combining (3.8) and (3.11), we obtain,

$$
\begin{aligned}
& n\left|a_{n}\right| \leq \sum_{t=1}^{p} 2 t\left|a_{t}\right|+\sum_{k=p+1}^{n-1}\left(\sum_{s=1}^{p} \frac{2 s(k+p) !}{(p+s) !(p-s) !(k-p-1) !\left(k^{2}-s^{2}\right)}\right. \\
&\left.\times\left(\sum_{t=1}^{s-1} 4 t\left|a_{t}\right|+2 s\left|a_{s}\right|\right)\right) \\
&+\sum_{s=1}^{p} \frac{2 s(n+p) !}{(p+s) !(p-s) !(n-p-1) !\left(n^{2}-s^{2}\right)}\left(\sum_{t=1}^{s-1} 2 t\left|a_{t}\right|+s\left|a_{s}\right|\right) .
\end{aligned}
$$

Therefore,

$$
\begin{aligned}
n\left|a_{n}\right| \leq & \sum_{t=1}^{p} 2 t\left|a_{t}\right| \\
& +\sum_{k=p+1}^{n-1} \sum_{s=2}^{p} \frac{2 s(k+p) !}{(p+s) !(p-s) !(k-p-1) !\left(k^{2}-s^{2}\right)} \sum_{t=1}^{s-1} 4 t\left|a_{t}\right| \\
& +\sum_{k=p+1}^{n-1} \sum_{s=1}^{p} \frac{4 s^{2}(k+p) !}{(p+s) !(p-s) !(k-p-1) !\left(k^{2}-s^{2}\right)}\left|a_{s}\right| \\
& +\sum_{s=2}^{p} \frac{2 s(n+p) !}{(p+s) !(p-s) !(n-p-1) !\left(n^{2}-s^{2}\right)} \sum_{t=1}^{s-1} 2 t\left|a_{t}\right| \\
& +\sum_{s=1}^{p} \frac{2 s^{2}(n+p) !}{(p+s) !(p-s) !(n-p-1) !\left(n^{2}-s^{2}\right)}\left|a_{s}\right| .
\end{aligned}
$$

Thus,

$$
\begin{aligned}
n\left|a_{n}\right| \leq & \sum_{t=1}^{p} 2 t\left|a_{t}\right| \\
& +\sum_{k=p+1}^{n-1} \sum_{t=1}^{p-1} \sum_{s=t+1}^{p} \frac{8 s(k+p) !}{(p+s) !(p-s) !(k-p-1) !\left(k^{2}-s^{2}\right)} t\left|a_{t}\right| \\
& +\sum_{k=p+1}^{n-1} \sum_{t=1}^{p} \frac{4 t^{2}(k+p) !}{(p+t) !(p-t) !(k-p-1) !\left(k^{2}-t^{2}\right)}\left|a_{t}\right| \\
& +\sum_{t=1}^{p-1} \sum_{s=t+1}^{p} \frac{4 s(n+p) !}{(p+s) !(p-s) !(n-p-1) !\left(n^{2}-s^{2}\right)} t\left|a_{t}\right| \\
& +\sum_{t=1}^{p} \frac{2 t^{2}(n+p) !}{(p+t) !(p-t) !(n-p-1) !\left(n^{2}-t^{2}\right)}\left|a_{t}\right| .
\end{aligned}
$$


Therefore,

$$
\begin{aligned}
n\left|a_{n}\right| \leq \sum_{t=1}^{p-1}[2+ & \sum_{k=p+1}^{n-1} \sum_{s=t+1}^{p} \frac{8 s(k+p) !}{(p+s) !(p-s) !(k-p-1) !\left(k^{2}-s^{2}\right)} \\
& +\sum_{k=p+1}^{n-1} \frac{4 t(k+p) !}{(p+t) !(p-t) !(k-p-1) !\left(k^{2}-t^{2}\right)} \\
& +\sum_{s=t+1}^{p} \frac{4 s(n+p) !}{(p+s) !(p-s) !(n-p-1) !\left(n^{2}-s^{2}\right)} \\
& +\left[2+\sum_{k=p+1}^{n-1} \frac{2 t(n+p) !}{(2 p) !(k-p-1) !\left(k^{2}-p^{2}\right)}\right] t\left|a_{t}\right| \\
& \left.+\frac{2 p(n+p) !}{(2 p) !(n-p-1) !\left(n^{2}-p^{2}\right)}\right] p\left|a_{p}\right| .
\end{aligned}
$$

Using Lemma 2, we obtain,

$$
\begin{aligned}
n\left|a_{n}\right| \leq & \sum_{t=1}^{p-1} \frac{2 n t(n+p) !}{(p+t) !(p-t) !(n-p-1) !\left(n^{2}-t^{2}\right)}\left|a_{t}\right| \\
& +\frac{2 n p(n+p) !}{(2 p) !(n-p-1) !\left(n^{2}-p^{2}\right)}\left|a_{p}\right|
\end{aligned}
$$

which is equivalent to (3.4).

Inequality (3.4) is known to be sharp for functions in $S(p)$ with real coefficients [2]. Since $S(p)$ is contained in $K(p),(3.4)$ is the best possible inequality that can be obtained for $K(p)$.

\section{REFERENCES}

[1] A. W. Goodman, On some determinants related to p-valent functions, Trans. Amer. Math. Soc., 63 (1948), 175-192.

[2] A. W. Goodman and M. S. Robertson, $A$ class of multivalent functions, Trans. Amer. Math. Soc., 70 (1951), 127-136.

[3] R. J. Leach, Coefficient estimates for certain multivalent functions, Pacific J. Math., 74 (1978), 133-142.

[4] R. J. Libera and E. J. Zlotkiewicz, Early coefficients of the inverse of a regular convex function, Proc. Amer. Math. Soc., 85 (1982), 225-230.

[5] A. E. Livingston, p-valent close-to-convex functions, Trans. Amer. Math. Soc., 115 (1965), 161-179. 
[6] The coefficients of multivalent close-to-convex functions, Proc. Amer. Math. Soc., 21 (1969), 545-552.

[7] M. S. Robertson, Multivalently star-like functions, Duke Math. J., 20 (1953), 539-550.

Received March 2, 1984 and in revised form May 7, 1984.

UNIVERSity OF Delaware

NEWARK, DE 19716 



\section{PACIFIC JOURNAL OF MATHEMATICS EDITORS}

V. S. VARAdarajan (Managing Editor)
University of California
Los Angeles, CA 90024
CHARLES R. DePrima
California Institute of Technology
Pasadena, CA 91125
R. FINN
Stanford University
Stanford, CA 94305

V. S. VARADARAJAN (Managing Editor)

Hermann FlaschKa

University of Arizona

Tucson, AZ 85721

RAMESH A. GANGOlli

University of Washington

Seattle, WA 98195

ROBION KIRBY

University of California

Berkeley, CA 94720
C. C. MOORE

University of California

Berkeley, CA 94720

H. SAMELSON

Stanford University

Stanford, CA 94305

Harold Stark

University of California, San Diego

La Jolla, CA 92093

\section{ASSOCIATE EDITORS}
R. ARENS
E. F. BECKENBACH
B. H. NeumanN
F. WOLF
K. Yoshida (1906-1982)

\section{SUPPORTING INSTITUTIONS}

\author{
UNIVERSITY OF ARIZONA \\ UNIVERSITY OF BRITISH COLUMBIA \\ CALIFORNIA INSTITUTE OF TECHNOLOGY \\ UNIVERSITY OF CALIFORNIA \\ MONTANA STATE UNIVERSITY \\ UNIVERSITY OF NEVADA, RENO \\ NEW MEXICO STATE UNIVERSITY \\ OREGON STATE UNIVERSITY
}

UNIVERSITY OF OREGON UNIVERSITY OF SOUTHERN CALIFORNIA

STANFORD UNIVERSITY

UNIVERSITY OF HAWAII

UNIVERSITY OF TOKYO

UNIVERSITY OF UTAH

WASHINGTON STATE UNIVERSITY

UNIVERSITY OF WASHINGTON 


\section{Pacific Journal of Mathematics}

\section{Vol. 120, No. $1 \quad$ September, 1985}

Ulrich F. Albrecht, A note on locally $A$-projective groups $\ldots \ldots \ldots \ldots \ldots 1$

Marilyn Breen, A Krasnosel'skiü-type theorem for unions of two starshaped

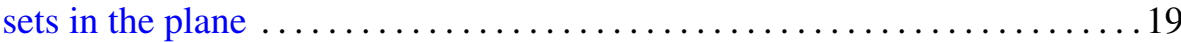

Anthony Carbery, Sun-Yung Alice Chang and John Brady Garnett,

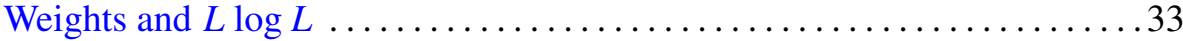

Joanne Marie Dombrowski, Tridiagonal matrix representations of cyclic

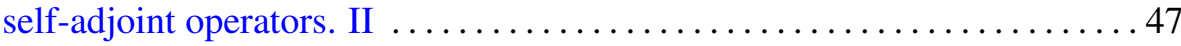

Heinz W. Engl and Werner Römisch, Approximate solutions of nonlinear random operator equations: convergence in distribution $\ldots \ldots \ldots \ldots \ldots 55$

P. Ghez, R. Lima and J. E. Roberts, $W^{*}$-categories $\ldots \ldots \ldots \ldots \ldots \ldots$

Barry E. Johnson, Continuity of homomorphisms of Banach $G$-modules . . 111 Elyahu Katz and Sidney Allen Morris, Free products of topological groups

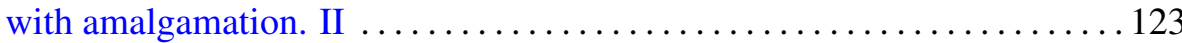

Neal I. Koblitz, $p$-adic integral transforms on compact subgroups of $\mathbf{C}_{p} \ldots 131$ Albert Edward Livingston, A coefficient inequality for functions of positive real part with an application to multivalent functions $\ldots \ldots \ldots 139$

Scott Carroll Metcalf, Finding a boundary for a Hilbert cube manifold

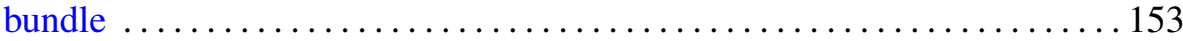

Jack Ray Porter and R. Grant Woods, When all semiregular $H$-closed extensions are compact ........................

Francisco José Ruiz and José Luis Torrea, A unified approach to Carleson measures and $A_{p}$ weights. II $\ldots \ldots \ldots \ldots \ldots \ldots \ldots \ldots \ldots \ldots \ldots \ldots \ldots$

Timothy DuWayne Sauer, The number of equations defining points in general position

John Brendan Sullivan, Universal observability and codimension one subgroups of Borel subgroups

Akihito Uchiyama, Extension of the Hardy-Littlewood-Fefferman-Stein inequality 